\title{
Discrimination syndicale en Turquie
}

L'action syndicale dans un régime semi-autoritaire

Anti-Union Discrimination in Turkey. Trade Union Action in a Semi-

Authoritarian Regime

Isil Erdinç

\section{(2) OpenEdition}

1 Journals

Édition électronique

URL : http://journals.openedition.org/travailemploi/7090

DOI : 10.4000/travailemploi.7090

ISSN : 1775-416X

Éditeur

DARES - Ministère du Travail

\section{Édition imprimée}

Date de publication : 1 avril 2016

Pagination : 101-123

ISSN : 0224-4365

\section{Référence électronique}

Isil Erdinç, « Discrimination syndicale en Turquie », Travail et Emploi [En ligne], 146 | avril-juin 2016, mis en ligne le 11 juillet 2019, consulté le 07 septembre 2019. URL : http://journals.openedition.org/ travailemploi/7090 ; DOI : 10.4000/travailemploi.7090 


\title{
Discrimination syndicale en Turquie
}

\section{L'action syndicale dans un régime semi-autoritaire"}

\author{
Isil Erdinç**
}

\begin{abstract}
Consacré à la période de gouvernement du Parti de la justice et du développement (Adalet ve Kalkınma Partisi, AKP) au pouvoir en Turquie depuis 2002, notre article étudie les pratiques discriminatoires anti-syndicales et les stratégies de résistance qui leur répondent. Il s'interroge sur la pérennisation de la discrimination syndicale, alors même que les réformes juridiques entreprises pour se conformer aux exigences européennes dans le cadre du processus d'adhésion à l'Union semblaient mettre le pays sur la voie de la démocratisation. À partir d'une enquête menée entre 2011 et 2015, consistant notamment en des observations ethnographiques dans les syndicats doublées d'une centaine d'entretiens semi-directifs auprès de dirigeants et permanents syndicaux, notre article analyse dans une première partie trois stratégies principales de discrimination syndicale mises en œuvre par le gouvernement, les employeurs et certains syndicats. Dans une seconde partie, il montre comment les syndicats luttent contre la discrimination, en s'appuyant sur des études de cas dans deux secteurs d'activité majeurs de l'économique turque : la métallurgie et les activités portuaires (construction navale et transport).
\end{abstract}

\footnotetext{
Ce travail étudie à la fois les pratiques discriminatoires mises en œuvre à l'encontre des syndicats sur les lieux de travail en Turquie et les stratégies de résistance possibles ${ }^{1}$, en se concentrant sur les trois mandats du gouvernement du Parti de la justice et du développement (Adalet ve Kalkınma Partisi ; AKP), depuis son arrivée au pouvoir en novembre 2002 jusqu'à sa quatrième réélection consécutive en novembre 2015.

* Merci à Sophie Béroud, Magali Boumaza, Benjamin Gourisse et Cem Özatalay pour leur relecture.

** Université Paris 1 Panthéon-Sorbonne, Centre européen de sociologie et de science politique-Centre de recherches politiques de la Sorbonne (CESSP-CRPS ; UMR 8209) ; isilerdinc2@ gmail.com.

1. La discrimination syndicale, telle que nous l'entendons dans cet article, caractérise toute pratique et réglementation juridique discriminatoire portant atteinte au droit des ouvriers de choisir librement leur syndicat. Promouvoir un syndicat plutôt qu'un autre compte également parmi les pratiques discriminatoires. La discrimination syndicale viole dans ce cas le principe d'égalité entre les organisations syndicales et entre les ouvriers syndiqués au sein de différentes organisations syndicales.
} 
Nous avons retenu la Turquie et la période du gouvernement AKP comme cadre d'étude car, dans cette économie néolibérale ${ }^{2}$ dotée d'un régime semi-autoritaire ${ }^{3}$, les pratiques anti-syndicales des employeurs vont de pair avec la répression politique des mobilisations syndicales ${ }^{4}$. Au cours des années 2000 , le processus d'adhésion à l'Union européenne (UE) marque à la fois la vie politique, économique et sociale et les réglementations juridiques en Turquie. Des pressions y sont exercées pour favoriser une libéralisation politique en général, et le respect des droits sociaux et syndicaux en particulier. Malheureusement, ces injonctions européennes, comme celles d'autres organisations internationales, sont allées de pair avec une ouverture économique qui a par ailleurs largement contribué à l'affaiblissement de la liberté syndicale. Dans un tel contexte, le rôle joué par l'Europe communautaire semble obéir à une logique paradoxale (ERDINÇ, VISIER, 2017). Les rapports de suivi de la Commission européenne sur les pays candidats montrent que, même lorsque les contraintes liées au droit communautaire sont censées peser, les libertés syndicales sont entravées et les ouvriers syndiqués insuffisamment protégés (COMMISSION EuROPÉENNE, 2013). S'agissant de la Turquie, comment expliquer la pérennisation de la discrimination syndicale alors que des réformes juridiques sont précisément adoptées pour démocratiser le pays ? Quels sont les mécanismes par lesquels s'exercent les politiques discriminatoires et quelles stratégies syndicales sont mises en œuvre pour lutter contre la discrimination syndicale ? Ce sont ces questions que nous abordons dans cet article.

Une multitude d'acteurs (gouvernementaux, entrepreneuriaux et syndicaux) ont recours à des pratiques discriminatoires, et forment une alliance de fait. Le droit social et la catégorie juridique de représentativité syndicale ${ }^{5}$ représentent pour eux des enjeux

2. Renvoyant aux travaux de Jamie Peck et d'Adam Tickell (PECK, Tickell, 2007), Cem ÖzATAlay précise que le néolibéralisme est passé de la phase « plus de marché, moins d'État », durant laquelle le néolibéralisme vise à réduire, voire à abolir les structures institutionnelles et sociales associées au keynésianisme, à celle où il a pour but de les réorganiser complètement (ÖZATALAY, 2010). L'influence du néolibéralisme sur la structure du pouvoir en Turquie ne s'est pas immédiatement fait sentir à la suite du coup d'État de 1980, comme on le croit généralement, mais à partir de la fin des années 1990 et plus particulièrement dans les années 2000. À cette période, le néolibéralisme a, lui aussi, changé de cap dans sa trajectoire (ÖZATALAY, 2010).

3. Les spécialistes de sciences politiques se sont souvent interrogés concernant le modèle que constitue le régime turc depuis les années 1980, et singulièrement sous le gouvernement AKP (BOZARSLAN, 2004 ; DorRonsOro, 2005 ; INSEL, 2008, 2015). Alors que la Constitution proclame que la république de Turquie est un État de droit, laïc, social (État providence) et démocratique, qui garantit les libertés d'association, de rassemblement et d'expression, les élections ne sont en réalité qu'une façade visant à assurer au régime une légitimité démocratique. En effet, les pratiques concrètes du pouvoir (notamment les arrestations de leaders et de militants des partis d'opposition et la censure de certains médias) rendent l'alternance politique pratiquement impossible. Bien que la notion de régime semi-autoritaire soit difficile à définir, il nous semble qu'elle correspond à la situation turque entre 2002 et 2015. Juan LiNZ (2006) définit l'autoritarisme comme un régime au pluralisme limité. Dans cet article, nous tenterons précisément de mettre en évidence les pratiques étatiques pouvant être qualifiées d'autoritaires.

4. Remonter au coup d'État militaire du 12 septembre 1980 permet de saisir pourquoi, à l'orée des années 2000, le syndicalisme a fait l'objet d'une violente répression politique en Turquie. Le coup d'État a conduit en effet à diminuer les droits sociaux et syndicaux. Le régime qui a alors pris le pouvoir a assuré l'intégration du pays au marché financier mondial et la mise en œuvre d'une politique néolibérale par des mesures autoritaires et de nouvelles réglementations juridiques empêchant la contestation sociale (TANÖR, YÜZBAşIOĞLU, 2005).

5. La représentativité signifie, selon le droit turc, être compétent au sein d'un secteur d'activité donné pour conclure des conventions collectives au niveau de l'entreprise (encadré 3). 
de première importance ${ }^{6}$. Ainsi, le ministère du Travail et de la Sécurité sociale, seule institution compétente dans la reconnaissance de la représentativité, fait un usage de la catégorie qui lui permet de renforcer son contrôle gouvernemental sur les syndicats. Les employeurs la mobilisent quant à eux afin de se protéger des syndicats les plus combatifs, en les empêchant de devenir représentatifs au sein de leur entreprise et/ou de leur secteur d'activité. Enfin, dans un contexte de forte concurrence pour recruter des adhérents, les syndicats font appel à cette notion pour renforcer leur position au sein du champ syndical. On voit alors se dessiner un système social qui se caractérise par des pratiques discriminatoires « sélectives », s'appliquant de façon inégale sur les syndicats issus des tendances de gauche (syndicats dits de contestation) et de droite (proches du gouvernement AKP). Des acteurs politiques, économiques et administratifs agissent de concert pour marginaliser les syndicats contestataires et promouvoir au contraire ceux qui sont proches du pouvoir. En réaction, les syndicats discriminés tentent de s'appuyer sur leurs ressources de terrain au niveau local ou de susciter des alliances à l'international. Cette analyse nous permet de réfléchir aux espaces possibles de la critique et de la contestation, quand les acteurs politiques nationaux tentent de mettre aux ordres certains acteurs syndicaux. Au-delà du thème de la discrimination, notre contribution rend également compte d'actions collectives qui, intervenant à l'échelle internationale, n'apparaissent pas pour autant déconnectées du local.

Jusqu'à présent, la question de la discrimination syndicale en Turquie a principalement été abordée en droit du travail (ÖZVERi, 2012) et en droit international à partir des analyses sur la conformité de la législation turque aux principes fondamentaux internationaux (GüLMEZ, 2005). La grande majorité des travaux, également en économie du travail, se focalise sur la violation des droits syndicaux ${ }^{7}$. En sciences politiques, l'analyse étudie principalement les stratégies partisanes et gouvernementales et se limite donc à l'aspect politique des discriminations syndicales. Les départements de recherche de certains syndicats, ainsi que des militants associatifs et politiques, ont commencé depuis quelques années à produire eux-mêmes des connaissances sur les discriminations et la répression syndicales ${ }^{8}$ (KAYGISIZ, 2012). À l'instar de ce qui s'est longtemps passé en France ${ }^{9}$, les recherches sur les discriminations syndicales reposent encore en Turquie sur un faible nombre d'observations et sur des méthodes indirectes.

Dans cet article, nous mobilisons précisément une enquête que nous avons menée entre décembre 2011 et novembre 2015 (encadré 1). La première partie de notre article

6. Ces questions se posent également en France (cf. par exemple le numéro que leur a consacré la revue Travail et Emploi [BÉroud et al., 2012] ou, de façon plus ancienne, l'article de Marc Pulvar [2002] sur la situation en Martinique).

7. Çelik Z. (2012), « Sendikal mücadelede yeni bir sayfa : Sendikalar ve Toplu İş Sözleşme Yasası » [Une nouvelle page dans la lutte syndicale : le code des syndicats et des conventions collectives] 21 octobre ; http://www.sendika. org/2012/10/sendikal-mucadelede-yeni-bir-sayfa-sendikalar-ve-toplu-is-sozlesme-yasasi-zeynep-celik/, consulté le 22 décembre 2016.

8. Öngel S. (2013), « Sendikalı olma demiyorum, hobi olarak yine ol », Birgün, 6 août.

9. Des travaux récents, tels qu'en rend compte par exemple le dernier numéro de Travail et Emploi ( $\left.{ }^{\circ}{ }^{145}\right)$, constituent de ce point de vue une inflexion notable. 
est consacrée à l'analyse du rôle des principaux acteurs mettant en œuvre des pratiques discriminatoires : le gouvernement AKP, l'administration, les employeurs et une partie des syndicats. Dans notre seconde partie, nous montrons comment les syndicats victimes de discriminations tentent d'y résister, en faisant appel soit aux instances européennes ou internationales, soit aux alliances interprofessionnelles locales. Pour ce faire, nous étudions plus particulièrement deux secteurs d'activité importants dans l'économie turque : la métallurgie (en prenant le cas des compagnies multinationales Renault et Bosch à Bursa dans le nord-ouest de l'Anatolie) et les activités portuaires (construction navale et transport aux docks de Tuzla à Istanbul). Nous avons choisi ces secteurs parce qu'ils sont représentatifs des discriminations syndicales correspondant à la violation du principe d'égalité entre syndicats d'un même secteur. Leur étude révèle une corrélation entre précarité, flexibilité, sous-traitance et discrimination syndicale. Les secteurs de la métallurgie, du transport maritime et du textile sont des secteurs précaires notamment parce qu'en leur sein les ouvriers risquent le licenciement s'ils n'adhèrent pas à un syndicat promu par les employeurs et par l'État (tableau 1, dans l'encadré 1).

\section{ENCADRÉ 1}

\section{Matériaux et sources}

Cet article s'appuie sur des matériaux recueillis dans le cadre d'un travail de terrain réalisé entre décembre 2011 et novembre 2015.

Nous avons mené 110 entretiens semi-directifs avec 55 dirigeants et 55 permanents syndicaux et confédéraux travaillant au sein des trois principales confédérations syndicales de travailleurs. Le corpus est ainsi composé de 15 permanents et 20 dirigeants de DİSK ${ }^{1}$, 15 permanents et 15 dirigeants de Türk-İş, 25 permanents et 20 dirigeants de Hak-İs. Nous avons également effectué des entretiens supplémentaires avec 4 dirigeants et 3 permanents de Memur-Sen, et 5 permanents de KESK.

Nous nous sommes par ailleurs livrée à une analyse de différents rapports et sources statistiques :

- rapports de suivi de l'Union européenne sur la Turquie publiés par la Commission européenne ;

- statistiques officielles du ministère turc du Travail et de la Sécurité sociale sur les conflits du travail ;

- rapports annuels sur les droits sociaux d'associations des droits de l'homme en Turquie ;

- rapports annuels de la Confédération syndicale internationale (CSI) sur la violation des droits syndicaux.

Nous avons agrégé ces données, ce qui nous a permis de calculer les pourcentages et la répartition sectorielle des discriminations syndicales et de construire les tableaux statistiques présentés dans cet article. En l'absence de chiffres officiels exhaustifs sur les

1. Cf. infra, p. 106, pour la présentation des différentes organisations syndicales turques. 
discriminations syndicales, nous avons ainsi suivi le conseil d'Étienne PENISSAT et de Karel YoN qui notent qu' « en dehors des chiffres produits par l'État et les instituts de sondage, il est possible pour les chercheurs de construire eux-mêmes des données sur des effectifs plus petits, par exemple dans le cadre d'études monographiques » (PENISSAT, Yon, 2014, p. 57).

\section{TABLEAU 1 - Répartition sectorielle des pratiques discriminatoires (recensées), des effectifs syndiqués et de l'ensemble des salariés}

\begin{tabular}{|c|c|c|c|c|c|c|c|c|c|c|}
\hline & & 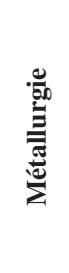 & 总 & 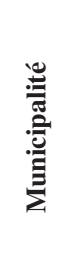 & 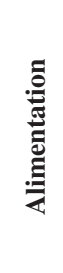 & 莧 & 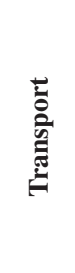 & 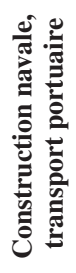 & 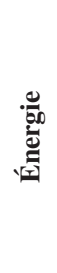 & 产 \\
\hline $\begin{array}{l}\text { Discriminations } \\
\text { syndicales }\end{array}$ & 30,5 & 15,5 & 14,0 & 13,0 & 8,4 & 7,0 & 7,0 & 2,3 & 2,3 & 100 \\
\hline Effectifs syndiqués & 13,5 & 32,8 & 1,1 & 21,7 & 10,6 & 5,7 & 6,2 & 1,5 & 6,8 & 100 \\
\hline Effectifs salariés & 18,4 & 25,4 & 5,1 & 13,6 & 9,6 & 8,5 & 12,6 & 2,7 & 4,1 & 100 \\
\hline
\end{tabular}

Note : étant donné qu'il n'existe pas de fichier recensant systématiquement les cas de discrimination déclarés, le tableau et les suivants ont été réalisés à partir des cas de discrimination relevés par l'auteure (courant 2013 et 2014), qui représentent environ $1 \%$ des ouvriers et des fonctionnaires syndiqués.

Note : en Turquie, le nombre total d'ouvriers est de 10881 618, dont 1001671 sont syndiqués (janvier 2013). Le nombre total de fonctionnaires est de 2134 638, dont 1468021 sont syndiqués (juillet 2013) selon les chiffres du ministère du Travail et de la Sécurité sociale. De façon plus précise, selon ces mêmes données, en juillet 2013, la répartition des effectifs syndiqués et salariés pour ces secteurs est la suivante : 87567 sur 1012566 (textile), 212443 sur 1396755 (métallurgie), 7419 sur 280842 (santé), 140895 sur 748069 (municipalité), 68706 sur 527153 (alimentation), 36942 sur 470178 (chimie), 40267 sur 693664 (transport), 10042 sur 147244 (construction navale, transport portuaire), 44016 sur 227950 (énergie).

Source : données calculées par l'auteure sur la base des rapports de suivi de la Commission européenne, de la Confédération syndicale internationale (CSI) et des associations de droits de l'homme.

Nous avons effectué par ailleurs des observations ethnographiques plus précises au sein même des syndicats et aux docks à Istanbul pour cartographier les pratiques discriminatoires et identifier les secteurs d'activité les plus touchés. À plusieurs reprises depuis 2011, lors de rencontres (formelles et informelles) à DİSK, Hak-İş et Türk-İss, et aux docks, nous avons recueilli les témoignages d'ouvriers syndiqués et de dirigeants syndicaux.

Nous avons de plus collecté de nombreux documents papier : tracts syndicaux, témoignages, rapports et statistiques produits par les instituts de recherche rattachés aux syndicats, lettres d'avertissement d'employeurs, articles de presse, etc. À la demande des permanents syndicaux chargés des relations internationales du Birleşik Metal-İş et de DİSK, nous avons traduit des documents officiels du turc vers le français et du français vers le turc, ce qui nous a notamment permis d'avoir accès aux textes utilisés par les syndicats en Turquie. Enfin, nous avons pu prendre connaissance des lettres de soutien que les syndicats français ont adressées en marque de solidarité à la direction de DİSK. 


\section{Pratiques discriminatoires : un jeu à multiples acteurs (gouvernement, patronat, syndicats)}

En Turquie, le syndicalisme est organisé par secteur d'activité ; par ailleurs, il y existe un pluralisme syndical qui, comme en France, correspond à des clivages politiques. Toutefois, l'existence autonome de syndicats régionaux, locaux ou d'entreprises est interdite. Le paysage syndical est ainsi seulement structuré aux niveaux sectoriel et national, par trois principales confédérations syndicales ouvrières et trois confédérations syndicales de fonctionnaires.

La Confédération des syndicats ouvriers de la Turquie (Türkiye İş̧i Sendikaları Konfederasyonu, Türk-İş), fondée en 1952, est la confédération la plus ancienne et rassemble le nombre le plus élevé d'ouvriers syndiqués. Elle se définit comme « suprapolitique », «non engagée » et « sans couleur [politique] » (Koç, 2010). La deuxième plus grande confédération syndicale, la Confédération des syndicats ouvriers réels (Hak Isşci Sendikaları Konfederasyonu, Hak-İş ${ }^{10}$ ) a été créée en 1976 par le mouvement islamique Milli Görǚş (Vision nationale). Elle se définit comme conservatrice. C'est de ce mouvement islamique que les principaux fondateurs de l'AKP sont issus. La Confédération des syndicats ouvriers révolutionnaires (Devrimci İşçi Sendikaları Konfederasyonu, DİSK), fondée en 1967 par des ouvriers et militants issus de différentes fractions de la gauche (socialiste, marxiste-léniniste, sociale-démocrate) est la troisième confédération syndicale ouvrière ${ }^{11}$.

Les mêmes lignes de partage se retrouvent dans le syndicalisme du secteur public. La Confédération des syndicats du secteur public de Turquie (Türkiye Kamu-Sen), de tendance nationaliste ${ }^{12}$, fondée en 1992, est la première confédération syndicale de fonctionnaires. La Confédération des syndicats des travailleurs du secteur public (Kamu Emekçileri Sendikaları Konfederasyonu, KESK), créée en 1995, est plutôt de gauche, socialiste. La Confédération des syndicats de fonctionnaires (Memur Sendikaları Konfederasyonu, Memur-Sen), également créée en 1995, se présente à l'inverse comme conservatrice et privilégiant des valeurs islamiques. Considérée comme proche de l'AKP, elle soutient les politiques gouvernementales depuis 2002.

L'histoire du syndicalisme en Turquie est marquée par le coup d'État militaire du 12 septembre 1980, mais aussi plus largement, comme dans de nombreux pays, par la crise du syndicalisme au cours des décennies 1980 et 1990. Au lendemain du coup d'État, des milliers de syndicalistes et de représentants ouvriers sont emprisonnés,

\footnotetext{
10. Le mot hak est polysémique ; il signifie à la fois «Dieu (Allah) », « droit », « juste », « vrai », « réel ». La traduction officielle du nom de la confédération en anglais est Confederation of Real Trade Unions.

11. Depuis le coup d'État militaire du 12 septembre 1980 qui a vu son positionnement se modifier (adhésion à la Confédération européenne des syndicats [CES] en 1985, renouveau des stratégies syndicales autour des questions européennes, de l'égalité entre les sexes, l'âge, etc.), DİSK est passée par plusieurs périodes de transformation. Aujourd'hui, alors que les dirigeants et permanents de certains syndicats qui lui sont affiliés se présentent toujours comme marxistes-léninistes, DİSK est plus proche des groupes sociaux-démocrates au niveau confédéral.

12. Défendant l'idée de l'unité de la nation turque, elle est particulièrement hostile à l'autonomie du mouvement kurde.
} 
tandis que des milliers d'ouvriers sont licenciés ou partent en exil. Certains syndicats affiliés à Türk-İş, alors même que la Confédération semble proche du pouvoir, font l'objet de suspensions qui durent entre quelques jours et deux ans. Hak-İş est interdite plusieurs mois en 1980 et 1981, DİSK l'étant jusqu'en 1992, de même que les syndicats qui lui sont affiliés. Plusieurs recherches sur le syndicalisme turc observent que la période du gouvernement AKP marque une nouvelle phase, particulièrement forte, de répression du syndicalisme. Elles présentent les années 2000 comme une période de recul pour le mouvement syndical, au cours de laquelle les ouvriers et les fonctionnaires ont été pris dans des rapports de force défavorables. La nette diminution de l'ampleur et de l'influence des manifestations syndicales serait due à la précarisation du marché du travail (BUĞRA, 2010 ; Koç, 2010).

\section{Discrimination syndicale et action étatique sous le gouvernement AKP}

Au cours des années 2000, le processus global de désyndicalisation en Turquie s'accompagne d'une tendance inverse, et spécifique, de croissance des syndicats privilégiés par le gouvernement $\mathrm{AKP}^{13}$. Plus largement, l'AKP s'implante dans l'État et met en place un système sélectif de redistribution des ressources publiques. La majorité parlementaire dont dispose l'AKP depuis 2002 lui permet de transformer l'État, d'appliquer des politiques néolibérales et de reconfigurer le fonctionnement des différents pans de l'économie et de la société (cette situation n'est pas exceptionnelle dans l'histoire turque, l'État étant resté particulièrement perméable aux forces politiques en présence depuis la proclamation de la République en 1923 ; GoURISSE, 2012). L'implantation de l'AKP au sein des structures étatiques se fait principalement par un recrutement sélectif dans l'administration, la justice et la police faisant partie des domaines les plus touchés (ATILGAN, SANCAR, 2009). Le gouvernement s'approprie ainsi l'appareil d'État, modifiant également la législation, voire la Constitution. Les marchés publics sont attribués aux entreprises qui lui sont proches, de même que les aides d'État (BALKAN, BALKAN, ÖNCÜ, 2010).

Nos observations montrent que l'action gouvernementale concernant le champ syndical a des conséquences indirectes sur la discrimination syndicale (encadré 2). Elle se décline selon nous en trois politiques stratégiques : la mise en place de nouvelles réglementations juridiques; l'utilisation de la violence et de la répression contre les syndicalistes de tendance socialiste, notamment ceux affiliés à DİSK et à KESK ; et le discours spécifique des ministres et du Premier ministre sur le « syndicalisme acceptable » (ÖZVERİ, 2012, pp. 254-255), qui vise à définir un syndicalisme légitime proche du parti au pouvoir, tel que celui d'Hak-İş et Memur-Sen.

S'inscrivant dans le cadre des réformes en vue de l'adhésion à l'Union européenne, le nouveau code des syndicats et des conventions collectives fait passer de $10 \%$ à $3 \%$ le taux de syndicalisation nécessaire pour être reconnu comme un syndicat représentatif

13. Çelik A. (2015), « Sendikalaşma gerçekten artıyor mu? » [Est-ce que le taux de syndicalisation augmente véritablement ?], T24, 27 janvier. 


\section{ENCADRÉ 2}

\section{Des pratiques anti-syndicales touchant très inégalement les organisations syndicales}

L'hétérogénéité des sources permettant de repérer les cas de discrimination syndicale est telle que nous ne pouvons pas affirmer que les données mobilisées dans l'article sont tout à fait représentatives. Elles nous paraissent cependant utiles, en ce qu'elles donnent une idée de la répartition des atteintes à la liberté syndicale selon les secteurs et des formes de discrimination, qui, comme nous l'avons observé, n'affectent pas tous les syndicats de la même manière.

Dans le secteur public, l'ensemble des cas déclarés concerne les syndicats affiliés à KESK, de tendance socialiste. Les syndicats de tendance islamo-conservatrice (affiliés à Memur-Sen) ou nationalistes (affiliés à la Türkiye Kamu-Sen) ne sont pas touchés. Dans le secteur privé, aucun cas de discrimination syndicale déclaré ne concerne un syndicat affilié à Hak-Işs, de tendance islamo-conservatrice, alors qu'ils se répartissent à parts égales entre Türk-İş (centriste-nationaliste) et DİSK (socialiste, sociale-démocrate). Les déséquilibres sont encore plus nets si l'on prend en compte le fait que Türk-İş rallie 69,4\% des ouvriers syndiqués, Hak-İş 16,4\% et DİSK seulement $10 \%$. Sous le gouvernement AKP, on est donc avant tout discriminé en fonction de son appartenance syndicale.

Selon les chiffres officiels du ministère du Travail et de la Sécurité sociale, le taux de syndicalisation est passé de 9,2\% à 10,7\% de 2013 à 2015 dans le secteur privé, et de 68,8\% à 70,3\% de 2012 à 2014 dans le secteur public. Cette augmentation est toutefois inégalement distribuée entre les trois principales confédérations syndicales. Dans le secteur privé, alors que $44 \%$ de cette augmentation concernent les syndicats affiliés à Hak-İş et $39 \%$ ceux affiliés à Türk-İş, seulement $17 \%$ peuvent être attribués aux syndicats de DİSK. Dans le secteur public, elle affecte à hauteur de $91 \%$ la Confédération des syndicats de fonctionnaires Memur-Sen, contre $5 \%$ pour la Confédération des syndicats du secteur public de la Turquie (Türkiye Kamu-Sen) et $3 \%$ KESK. En l'absence d'une réglementation juridique prévoyant la représentation égalitaire des confédérations, Türk-İş et Memur-Sen, qui regroupent la majorité des travailleurs syndiqués, sont les seules organisations reconnues représentatives dans certaines instances tripartites : Memur-Sen lors des négociations collectives relatives à la majorité des domaines relevant du secteur public et Türk-İşau sein de la commission de fixation du salaire minimum dans le secteur privé.

dans le secteur d'activité concerné (ÇALIŞMa ve Sosyal GÜVEnLIK BAKANLIĞI [Ministère du Travail et de la Sécurité sociale], 2012 ; encadré 3 pour une description du cadre légal). Cet assouplissement des critères de représentativité a toutefois des conséquences paradoxales pour le syndicalisme. Plusieurs clauses provisoires inscrites dans la loi éclairent les stratégies d'intervention du gouvernement AKP, qui a profité de l'évolution du cadre légal pour favoriser la représentativité de syndicats faiblement implantés mais qui lui sont proches, notamment ceux affiliés à Hak-İş. En plus de seuils transitoires de $1 \%$ et $2 \%$ appliqués en 2016 et 2017 (les $3 \%$ n'entrant en vigueur qu'au $1^{\text {er }}$ janvier 2018), deux exceptions notables aux nouvelles règles de représentativité se traduisent concrètement par une discrimination syndicale inversée. 
Premièrement, dans les trois premiers mois suivant l'entrée en vigueur de la loi en 2012, les syndicats fondés entre 2009 et septembre 2012 sont dispensés de l'obligation d'atteindre le seuil des $3 \%$ au niveau national : pour obtenir le droit de signer des conventions collectives, ils ne doivent dépasser les $3 \%$ qu'au niveau de l'entreprise. Deuxièmement, les syndicats qui bénéficiaient déjà du droit de signer des conventions collectives en juillet 2009, mais qui risquent de le perdre lors de la publication des nouvelles statistiques sur la représentativité syndicale en janvier 2013, la conservent pour la signature de leur prochaine convention collective. Selon Zeynep Çelik ${ }^{14}$, les clauses provisoires applicables aux syndicats créés à partir de 2009 concernent directement les nouveaux syndicats affiliés à Hak-İs : «L'abrogation du barrage pour les syndicats fondés après 2009 a été proposée par Hak-İs. Cela révèle le soutien de l'AKP aux syndicats de Hak-İş qui étaient en train de s'organiser au sein de l'Agence de presse Anatolie, du ministère de la Culture et dans plusieurs institutions publiques. Le gouvernement AKP voulait leur octroyer du temps pour qu'ils puissent faire adhérer plus d'employés en vue d'obtenir la représentativité et la majorité syndicale ${ }^{15}$.» Hak-İş se lance de fait dans un processus de syndicalisation à partir de 2007 et l'accélère entre 2009 et 2012, avec la fondation de plusieurs nouveaux syndicats affiliés dans les secteurs de la communication, du transport, des finances, de la santé, de la défense et de la papeterie. Ces syndicats ont donc directement profité de la clause introduite par le gouvernement. L'appui indirect dont ils ont bénéficié peut donc être qualifié de stratégie discriminatoire étatique.

\section{ENCADRÉ 3}

\section{Cadre juridique sur la représentativité et la discrimination syndicale en Turquie}

D'après le droit syndical turc, amendé en 2012 après son entrée en vigueur en 1983, l'obtention de la représentativité, et donc de la capacité à signer des conventions collectives au sein des entreprises, doit être effective à deux niveaux. D'une part, les syndicats doivent recueillir l'adhésion de plus de la moitié des ouvriers de l'entreprise. D'autre part, le nombre d'adhérents du syndicat doit permettre de dépasser un seuil national, qui est calculé à partir du nombre total des ouvriers travaillant au sein du secteur d'activité concerné. Les effectifs des syndicats sont calculés par le ministère du Travail et de la Sécurité sociale et rendus publics deux fois par an, en janvier et en juillet.

Trois sources principales encadrent le droit à la syndicalisation des salariés en Turquie : le droit interne turc, les contraintes juridiques du processus d'adhésion à l'Union européenne et les conventions de l'Organisation internationale du travail (OIT). Le droit turc a été amendé à plusieurs reprises pour aller dans le sens des conventions de l'OIT et des réformes européennes. La convention $n^{\circ} 98$ de 1949 sur le droit d'organisation et de

14. Çelik Z. (2012), « Sendikal mücadelede yeni bir sayfa : Sendikalar ve Toplu İş Sözleşme Yasası » [Une nouvelle page dans la lutte syndicale : le code des syndicats et des conventions collectives], art. cité (cf. note 7).

15. Citation traduite par l'auteure. 
négociation collective a été ratifiée par la Turquie en 1952. L'article 1 de la convention n ${ }^{\circ} 135$ de 1971 concernant les représentants des travailleurs, ratifiée par la Turquie en 1993, garantit la protection des représentants syndicaux contre les licenciements pour raisons syndicales. L'article 4 de la convention $n^{\circ} 151$ de 1978 sur les relations de travail dans la fonction publique, ratifiée par la Turquie également en 1993, protège les fonctionnaires contre les discriminations syndicales. Cependant, ces conventions n'ont été introduites dans la législation interne qu'à partir de 1992 avec le processus de l'élargissement de l'Union européenne. Dans le droit turc, la question de la discrimination syndicale est abordée principalement dans l'article 25 de la loi $n^{\circ} 6356$ sur les syndicats et les conventions collectives des ouvriers et dans l'article 18 de la loi $\mathrm{n}^{\circ} 4688$ sur les syndicats et les conventions collectives des fonctionnaires, également mis en vigueur en 2012. L'article 25 stipule ainsi : «L'embauche des ouvriers ne peut pas être conditionnée à l'adhésion à un syndicat ou à la démission d'un syndicat. L'employeur ne peut pas faire de discrimination entre les ouvriers syndiqués et les non-syndiqués, s'agissant des conditions de travail et des licenciements. » Le principe d'égalité entre syndicats, quant à lui, ne se trouve pas dans la législation turque.

Outre le droit syndical, le gouvernement AKP a également recours au droit pénal, à la répression d'État et à la violence policière pour peser dans le champ syndical. Il définit certaines activités syndicales comme illégales et agit au niveau individuel en procédant à l'arrestation de syndicalistes et d'ouvriers syndiqués. Des membres de Dev Sağlık-İş dans le secteur de la santé ${ }^{16}$, de Nakliyat-İş dans le secteur des transports ${ }^{17}$ et d'Enerji-Sen ${ }^{18}$ dans celui de l'énergie, tous affiliés à DİSK, sont ainsi arrêtés pour des raisons liées à leurs activités syndicales en 2012, 2013 et 2014. Douze syndicalistes de la DİSK, y compris le président et le secrétaire général, sont appréhendés ${ }^{19}$. Dirigeants syndicaux et ouvriers syndiqués sont accusés d'avoir participé à des manifestations

16. En janvier 2013, alors que Dev-Sağlık İş s'est lancé dans un processus de mobilisation et de syndicalisation des ouvriers contractuels qui, d'après la loi, n'ont pas le droit d'adhérer à un syndicat, une manifestation qu'il a organisée est interrompue par la police et vingt-sept ouvriers et dirigeants syndicaux, dont la présidente de Dev-Sağlık İş et la secrétaire générale de DİSK, sont mis en garde à vue (« Taşeron protestosuna polis saldırısı » [Attaque de la police dans la manifestation contre la sous-traitance], Birgün, 23 janvier 2013).

17. En février 2013, la compagnie postale Yurtiçi Kargo licencie soixante ouvriers ayant manifesté leur volonté d'adhérer au syndicat Nakliyat-İş. Vingt-cinq ouvriers et dirigeants syndicaux, y compris le président de Nakliyat-İş et le président adjoint de DİSK de l'époque, sont mis en garde à vue par la police alors qu'ils protestaient à Istanbul contre les licenciements ( « Nakliyat-İş sendikası üyesi 25 kişi gözaltına alındı » [25 membres du Nakliyat-İş en garde à vue], Özgür Gündem, 19 février 2013).

18. En août 2013, 540 ouvriers de Boğaziçi Elektrik Dağıtım Anonim Şirketi (BEDAŞ), société anonyme de distribution d'électricité Boğaziçi, sont licenciés. Une manifestation devant le bureau central du BEDAŞ, à Taksim à Istanbul, se solde par trois ouvriers blessés ; les dirigeants syndicaux de l'Enerji-Sen de DİSK sont hospitalisés, victimes des émanations de gaz lacrymogène et des coups de matraque de la police. Onze ouvriers, le président et l'ancien président de l'Enerji-Sen sont mis en garde à vue («Enerji-Sen üyelerine polis müdahalesi » [Intervention policière sur les membres d'Enerji-Sen] ; http://www.demokrathaber.net/calisma-hayati/enerji-sen-uyelerine-polis-mudahalesih21575.html, consulté le 30 novembre 2016).

19. Confédération syndicale internationale (CSI) (2013), "Turkey: More Than 100 Trade Unionists Arrested" ; http://www.ituc-csi.org/turkey-more-than-100-trade, consulté le 22 décembre 2016. 
interdites, opposé résistance aux forces policières, troublé la paix, la sécurité et l'ordre public et adhéré à des organisations portant atteinte à l'ordre public et à l'unité nationale.

Le processus de discrimination syndicale que le gouvernement AKP met en œuvre est également présent au niveau discursif. Ce qui relève du « bon syndicalisme » ou, à l'inverse, d'un « syndicalisme marginal » est défini par plusieurs critères régulièrement mobilisés dans les discours des ministres et de l'ancien Premier ministre Erdoğan, désormais président de la République ${ }^{20}$. Le discours de Bülent Arınç, vice-Premier ministre du gouvernement AKP, au congrès national de Hak-İş en octobre 2013 liste les fondements du bon syndicalisme :

« Hak-İş est un très bon syndicat et un très grand don du ciel pour la Turquie. Ils aiment leur nation, leur pays, leur drapeau, leur sol, leur peuple, leurs employés. Ils ne sont pas un syndicat marginal, idéologique. Ils cherchent leurs droits dans le cadre des contraintes de notre pays et des méthodes démocratiques. Ils ont toujours été honnêtes dans leurs relations avec les employeurs. C'est pourquoi ils ont toujours augmenté le nombre de leurs adhérents et, grâce soit rendue à Dieu, ils sont devenus l'un des syndicats les plus représentatifs aujourd'hui ${ }^{21}$. »

L'affichage des valeurs nationales (amour du pays, unité nationale, identité turque-musulmane-sunnite) et des valeurs morales (coutumes, famille, fraternité) est également l'un des indicateurs qui permet de qualifier un syndicaliste de «bon syndicaliste ». Ce discours en faveur des syndicats affiliés à Hak-İş et Memur-Sen se concrétise par des avantages juridiques (cf. supra) et légitime la répression d'État et la violence policière à l'encontre des syndicats affiliés à DİSK et KESK.

L'analyse des seules activités gouvernementales ne suffit pas pour comprendre le renforcement des pratiques discriminatoires. Les politiques gouvernementales au niveau national s'accompagnent de stratégies patronales au niveau local et au niveau de l'entreprise. Celles-ci sont observables dans le secteur privé comme dans le secteur public.

\section{Stratégies discriminatoires patronales}

Dans la lignée des politiques gouvernementales de reconstruction du champ syndical, les employeurs font la promotion des syndicats affiliés à Hak-İş et parfois à Türk-İş, et stigmatisent au contraire les employés adhérant aux syndicats affiliés à DİSK et KESK (ÖZvERİ, 2012). Les stratégies discriminatoires sont diverses. Selon

20. Il est possible de renvoyer ici à la notion d' « entrepreneurs de morale » d'Howard BECKER (1985) dans la manière dont le gouvernement AKP définit les syndicats marginaux. Les normes et les valeurs deviennent un enjeu politique et finalement un enjeu du champ syndical. Le gouvernement AKP joue le rôle d' « entrepreneur de morale » en construisant de nouvelles normes et en distinguant ce qui est « normal » ou « acceptable » de ce qui est « marginal » ou « déviant ». 21. «Hak-İş’ten 38. yıla görkemli kutlama » [Grandes célébrations pour le $38^{\mathrm{e}}$ anniversaire de Hak-İş], 25 octobre 2013, compte rendu du congrès national. 
le rapport annuel de l'Association des droits sociaux ${ }^{22}$ en 2012, la majorité des cas déclarés de discriminations correspondent au licenciement abusif d'ouvriers pour raisons syndicales (Sosyal HaKLAR DerneĞI, 2012). Les modifications de tâches ou de responsabilités des ouvriers, les suspensions de contrat de travail, et même les arrestations $^{23}$ et violences physiques comptent également parmi les pratiques observées (tableau 2).

TABLEAU 2 - Principales formes des pratiques discriminatoires patronales (recensement par des observateurs nationaux et internationaux)

\begin{tabular}{lcccccc}
\hline & Licenciement & $\begin{array}{c}\text { Mutation } \\
\text { forcée }\end{array}$ & $\begin{array}{c}\text { Violence } \\
\text { physique }\end{array}$ & Arrestation & $\begin{array}{c}\text { Suspension } \\
\text { du contrat } \\
\text { de travail }\end{array}$ & $\begin{array}{c}\text { Modification } \\
\text { des tâches et des } \\
\text { responsabilités }\end{array}$ \\
\hline Secteur privé & 83,0 & 9,0 & 2,3 & 2,3 & 2,3 & 1,1 \\
Secteur public & 24,0 & 24,0 & 20,0 & 16,0 & 12,0 & 4,0 \\
\hline
\end{tabular}

* Il s'agit de mutations de fonctionnaires d'un lieu d'exercice à un autre (changement de ville, de département, de région).

Source : données calculées par l'auteure sur la base des rapports de suivi de la Commission européenne, de la CSI et des associations de droits de l'homme.

Que ce soit au sein des entreprises privées, publiques ou parapubliques, forcer des travailleurs à adhérer à des syndicats de Hak-İş ressort comme une pratique discriminatoire courante. Ainsi, en 2013, les ouvriers travaillant au sein de l'entreprise publique de production de thé Çaykur (membre du syndicat du secteur de la production alimentaire de Türk-Iş, Tek Gida-İ̧s) ont été contraints d'adhérer au syndicat du secteur de la production alimentaire de Hak-İss, Öz Gıda-İss, sous peine de licenciement. Le secrétaire général de Türk-İş explique :

«Ils ont éloigné les ouvriers de notre syndicat. C'était Tek Gıda-İş qui était représentatif au niveau de l'entreprise, mais ils ont menacé nos ouvriers. Les responsables gouvernementaux eux-mêmes ont fait des déclarations soutenant Öz Gida-İş, ce qui n'est finalement pas choquant. Certains ouvriers ont changé de syndicat ; donc nous avons perdu la représentativité. »

$$
\begin{aligned}
& \text { (Entretien avec Mustafa Akyürek, } \\
& \text { secrétaire général du Tek Gıda-İş, } 22 \text { août } 2014^{24} \text { ) }
\end{aligned}
$$

Le fait que les ouvriers travaillant dans des municipalités tenues par l'AKP, notamment la municipalité d'Istanbul, aient été astreints à rejoindre le syndicat des ouvriers municipaux de Hizmet-İs, affilié à Hak-İş, est souvent mis en avant dans les rapports sur la violation des droits et des libertés syndicales publiés par la

22. L'Association des droits sociaux de Turquie (Sosyal Haklar Derneği) est créée en 2005-2006 par un groupe de militants de tendance socialiste et sociale-démocrate. Elle a pour objectif de recenser les cas de violation des droits sociaux, décrits dans les bilans de droits sociaux qu'elle publie.

23. Les employeurs font appel à la police ou bien portent plainte pour que les syndicalistes soient mis en garde à vue.

24. Tous les entretiens ont été réalisés par l'auteure. 
Confédération syndicale internationale $(\mathrm{CSI})^{25}$. Un permanent syndical au syndicat des ouvriers municipaux de DİSK, Genel-İş, explique :

« Ici, il s'agit de l'adhésion des ouvriers au syndicat affilié à Hak-İş. Quand la municipalité a déclaré qu'elle n'allait pas négocier avec les ouvriers affiliés aux autres confédérations en vue de signer des conventions collectives, les ouvriers ont été obligés de changer de syndicat pour pouvoir être couverts par une convention collective. »

(Entretien avec un permanent syndical du Genel-İş à DİSK, 19 septembre 2012)

Il existe également d'autres transferts d'employés vers les syndicats affiliés à Hak-İş fondés entre 2008 et 2013 comme Öz Orman-İş au sein de la Direction générale des forêts, Öz Büro-İş au sein du ministère de la Culture et du Tourisme ${ }^{26}$ et Öz Finans-İş, au sein des banques partiellement publiques comme Halkbank et Ziraat Bankasi $^{27}$.

Dans le secteur public, d' après nos observations, c'est souvent parce qu'ils savent qu'ils seront discriminés s'ils rejoignent un autre syndicat que Memur-Sen, que les travailleurs choisissent de s'y syndiquer. D' après notre enquête ${ }^{28}$, les principaux motifs d'adhésion à un syndicat affilié à Memur-Sen sont les suivants : le syndicat est plus « apte à obtenir plus d'avantages matériels de l'État », a « de bonnes relations avec le gouvernement AKP » et, en cas d'adhésion, « [il n'y a pas de] risque d'être arrêté ou licencié pour raisons syndicales ». Licenciement et mutation sont des risques qui pèsent principalement sur des fonctionnaires affiliés à KESK. Selon le rapport annuel de l'Association des droits sociaux de 2012, $48 \%$ des cas déclarés de pratiques discriminatoires correspondent à des licenciements et à des mutations liés à une affiliation syndicale à KESK ou à la participation aux activités syndicales et manifestations organisées par ce syndicat.

Une autre stratégie de discrimination syndicale menée par le patronat est liée au remplacement en 2012 du code des syndicats $n^{\circ} 2821$, alors en vigueur depuis 1983 , par un nouveau code ( $n^{\circ}$ 6356). L'article 22 de l'ancien code imposait aux ouvriers de passer par un notaire pour obtenir une attestation de démission d'un syndicat et d'adhésion à un autre. En 2012, cette obligation est supprimée et un système électronique est mis en place, qui a fragilisé les ouvriers face aux employeurs. Une dirigeante syndicale de DİSK explique comment, selon les témoignages des ouvriers, l'employeur confisque leur mot de passe pour les faire adhérer au syndicat que lui préfère :

« Les ouvriers, n'ayant pas accès à Internet ou ne connaissant pas le système électronique, adhèrent à un syndicat avec l'aide d'un dirigeant ou d'un représentant syndical,

25. CSI (2012), "Intimidation of public sector trade unionists" ; http://survey.ituc-csi.org/Intimidation-of-publicsector.html?lang=en, consulté le 22 décembre 2016.

26. Savaş A. (2013), « Türk-İş’ten kaçarken Hak-İş'e yakalanmak » [S'échapper de Türk-İş, se trouver à Hak-İş], Sol, 19 février.

27. Koç Y. (2013), « Sendikal operasyonda sıra bankalarda » [C'est au tour des banques dans l'opération syndicale], Sol, 24 août.

28. Entretien avec un dirigeant syndical de Memur-Sen, 21 août 2013. 
ou encore en présence de l'employeur. La majorité des employeurs gardent les mots de passe des ouvriers pour être sûrs qu'ils n'adhèrent pas à un syndicat sans leur avis. Ils laissent les dirigeants d'autres syndicats prendre les mots de passe des ouvriers pour les faire adhérer à leur syndicat. »

(Entretien avec une dirigeante syndicale de DİSK, 27 juin 2013)

Ainsi, les employeurs s'appuient sur les réglementations juridiques pour élaborer de nouvelles stratégies. Ils participent aux processus de marginalisation et de criminalisation des ouvriers et syndicats affiliés à DISSK et KESK, et à la promotion des syndicats affiliés à Hak-İş et Memur-Sen. La précarisation des secteurs privé et public expose les ouvriers et les fonctionnaires au risque de licenciement, de mutation, d'enquêtes disciplinaires internes, voire de violence physique. Nous avons de plus observé que les stratégies patronales sont soutenues par certains syndicats, dans un contexte de concurrence forte entre eux.

\section{Concurrence syndicale et discriminations}

Le déséquilibre entre organisations syndicales augmente pour plusieurs raisons : nous l'avons vu, les politiques gouvernementales et les pratiques discriminatoires patronales y contribuent. C'est également le cas de la précarité du marché du travail. Dans son rapport portant sur le secteur public, İrfan KAYGISIZ (2012) met en avant les effets de la fragilisation de la sécurité de l'emploi sur les droits et libertés syndicales. Dans un contexte de crise économique, des logiques managériales ont été mises en œuvre. Elles visent à réduire le déficit budgétaire, l'endettement et les dépenses publiques et s'appuient sur l'évaluation, l'efficacité des fonctionnaires et la rentabilité des institutions publiques. Dans ce cadre, la mutation d'un fonctionnaire jugé inefficace est devenue une pratique courante, ce qui a des effets sur les processus de syndicalisation (KAYGISIZ, 2012).

De plus, pour parvenir à recruter davantage d'adhérents alors que la concurrence est forte au sein du champ syndical, certains syndicats n'hésitent pas à prendre part aux pratiques discriminatoires mises en œuvre à l'encontre de syndicats rivaux. Hak-İş, Memur-Sen et certains syndicats affiliés à Türk-İş participent ainsi à la discrimination syndicale dont sont victimes DİSK, KESK et certains autres syndicats de Türk-İş ${ }^{29}$. Comme nous l'avons indiqué, il n'existe pas en Turquie de relevé systématique de ces pratiques, que ce soit par les syndicats ou par les institutions judiciaires. De fait, ce sont les rapports de l'Association des droits sociaux (Sosyal Haklar Derneği), les rapports de la CSI et de la Commission européenne et nos entretiens avec les ouvriers et des représentants syndicaux qui nous permettent de mettre en évidence la participation des syndicats aux pratiques discriminatoires. Dans ce qui suit, nous évoquons ainsi

29. Türk-İ̧s est en effet une confédération syndicale assez hétérogène, regroupant des syndicats issus de tendances politiques différentes qui s'affrontent parfois en son sein. 
trois cas de discrimination au sein des secteurs public et privé à partir de témoignages de fonctionnaires et d'ouvriers.

En mars 2010, des dirigeants du syndicat du secteur de la santé, Sağlık-Sen, affilié à Memur-Sen, proche du gouvernement AKP, ont menacé de licenciement, voire de violence physique, s'ils n'adhéraient pas à leur syndicat, des fonctionnaires d'un hôpital situé à Ağrı au sud de la Turquie, syndiqués au SES (Sağlık Emekçileri Sendikası ; syndicat du secteur de la santé de KESK) (COMMISSION EUROPÉENNE, 2013).

Plus haut dans cet article, nous avons évoqué comment le patronat se saisissait des changements introduits par le nouveau code des syndicats de 2012 pour forcer leurs employés à adhérer à tel ou tel syndicat. Avec l'ancien code et l'obligation de passer par un notaire pour obtenir des attestations de démission et d'adhésion, certains syndicats utilisaient des moyens de pression pouvant aller jusqu'à de la violence physique. Un permanent syndical de DİSK explique :

« Il y a un an, ils [des militants d'un syndicat concurrent de DİSK] ont kidnappé le fils du notaire pour le menacer. Ils voulaient l'empêcher de signer le document nécessaire pour les ouvriers qui voulaient changer de syndicat. Finalement, il n'a pas fait l'attestation et les ouvriers n'ont pas su quoi faire. Ils ont dû rester dans l'autre syndicat, même s'ils voulaient adhérer à notre syndicat. »

(Entretien avec un permanent syndical de DİSK, 15 février 2012)

Un ouvrier, syndiqué de Birleşik Metal-İş, travaillant dans le secteur de la métallurgie, décrit comment les syndicats participent à la répression syndicale au niveau de l'entreprise :

« Ils ont envoyé à chacun de nous dans l'usine une lettre officielle de la compagnie, cosignée par le syndicat, nous indiquant de quitter immédiatement notre syndicat si nous pensions à notre famille, à nos enfants. Ils ont organisé des réunions syndicales auxquelles nous étions obligés de participer parce que le patron nous l'avait demandé. Ils sont venus en plein milieu de notre journée de travail et nous ont demandé d'adhérer à leur syndicat en présence des patrons. »

(Témoignage écrit d'un ouvrier de Birleşik Metal-İş, réalisé le 30 mai 2012)

Certains syndicats, tels Hak-İş et Türk-İş, font ainsi partie des acteurs qui participent aux pratiques discriminatoires et prennent part à la violation des libertés syndicales des ouvriers. Les ouvriers et fonctionnaires syndiqués, ou qui souhaitent le devenir, sont exposés aux contraintes juridiques, politiques et économiques qui de fait fragilisent les droits syndicaux et de l'emploi. Ainsi marginalisés et/ou discriminés, les acteurs syndicaux sont amenés à réfléchir à de nouvelles stratégies pour lutter contre la discrimination syndicale. Le recours à l'international et le recours au local sont deux manières de s'opposer que nous avons observées dans notre enquête. 


\section{Résister aux discriminations syndicales}

Étudiant les stratégies mises en œuvre pour lutter contre les discriminations syndicales, nous avons pu noter que les entreprises concernées étaient de taille diverse, de dimension internationale, nationale ou locale, et intervenaient dans plusieurs secteurs d'activité. Dans ce cadre, les ressources dont disposent les acteurs syndicaux sont variées. La capacité à faire appel à des appuis étrangers varie notamment selon les syndicats et les secteurs d'activité. Le syndicat de la métallurgie de DİSK, Birleşik Metal-İs, a davantage de contacts avec des syndicats à l'étranger que Limter-İs, son homologue du transport portuaire et de la construction navale, dont les possibilités de mobiliser les réseaux internationaux sont moindres. Par conséquent, Limter-İ̧ a recours aux acteurs locaux et crée des alliances interprofessionnelles, en particulier avec les partis politiques. Dans ce qui suit, nous analysons deux niveaux de lutte possibles pour les syndicats - l'international et le local - ainsi que les modalités de cette mobilisation syndicale dans un contexte de fortes contraintes politiques, syndicales et économiques (cf. supra), dues aux transformations de l'économie et du marché du travail. Par ces deux études de cas, nous cherchons à expliquer comment les recours internationaux contre la discrimination syndicale (cas de Renault et de Bosch à Bursa) et celui aux alliances locales (exemple de la zone du transport portuaire et de la construction navale à Tuzla à Istanbul) permettent de résister aux pratiques discriminatoires.

\section{Le recours à l'international : les mobilisations syndicales de Renault et Bosch à Bursa}

Mettant en avant les processus de diffusion par lesquels s'internationalisent les idées et pratiques d'un mouvement social, Donatella della PORTA et Sidney TARROW (2005, p. 2-3) mentionnent les mécanismes d'externalisation dans lesquels des institutions supranationales sont sollicitées pour intervenir dans des problèmes ou conflits domestiques (SIMÉANT, 2009). À partir des années 1990, et notamment dans le cadre du processus d'adhésion à l'Union européenne (ÖNGÜN, 2009), les syndicats turcs mobilisent de plus en plus leurs contacts étrangers dans le but de faire pression sur le pouvoir politique national. Ils font ainsi appel à des institutions internationales, telles que l'OIT ou la Commission européenne, et aux organisations syndicales européennes et internationales comme la Confédération européenne des syndicats (CES), la Confédération syndicale internationale (CSI) et les syndicats des différents pays européens.

À Bursa, l'une des villes les plus industrialisées de la Turquie, le textile, l'automobile et la métallurgie sont les secteurs économiques les plus développés. Les syndicats de la métallurgie y sont particulièrement puissants et la concurrence fait rage entre le syndicat de DİSK, Birleşik Metal-İş, et celui de Türk-İss, Türk Metal.

En avril 2012, le président de DİSK, Erol Ekici, et le président adjoint, Ali Riza Küçükosmanoğlu, expliquent sur le site Emek Dünyası que « les ouvriers qui voulaient 
changer de syndicat en démissionnant du Türk Metal afin d'adhérer à Birleşik Metal-İş, ont été agressés physiquement par un groupe incluant le secrétaire général de Türk $\operatorname{Metal}^{30} \gg$. De fait, en novembre 2012, les ouvriers de Bosch participent à une manifestation organisée par des ouvriers de Renault à la suite d'un désaccord intervenu lors des négociations d'une convention collective par Türk Metal. Le président du Birleşik Metal-İş, Adnan Serdaroğlu, signale par la suite que les ouvriers de Bosch ont été menacés par un groupe armé de couteaux et de matraques, dans lequel se trouvaient non seulement des syndicalistes de Türk Metal, mais aussi le président adjoint du syndicat et le président de sa branche de Bursa.

Dans ses travaux sur les syndicalistes européens et l'internationalisation des militants syndicaux, Anne-Catherine WAGNER note qu' au sein des grandes entreprises, les syndicalistes mobilisent des ressources sociales, culturelles et linguistiques spécifiques, construisant ainsi un « capital militant international » (autrement dit, des dispositions et des savoir-faire internationaux) (WAGNER, 2004,p. 13). C'est le cas des permanents syndicaux chargés des relations internationales du Birleşik Metal-İş ${ }^{31}$ et de DİSK ${ }^{32}$, qui ont relayé les incidents relatés ci-dessus au niveau international. Ayant suivi leurs études secondaires dans des lycées anglophones de Turquie et diplômés de l'université, ils sont dotés d'un capital linguistique leur permettant de construire des liens avec d'autres syndicalistes européens. Ils ont ainsi rédigé en anglais des rapports dans lesquels ils exposent à leurs collègues syndiqués étrangers les arguments et preuves présentés au tribunal du travail et à la Cour de cassation turcs, puisque l'affaire de Bursa a été portée en justice. Ils ont notamment tenu au courant les syndicats français et allemands, en particulier les syndicats représentatifs chez Renault et chez Bosch, de l'avancement des procès. Afin de proposer un dossier le plus complet possible, ils ont traduit des documents juridiques, les arrêts intermédiaires des procès, des déclarations officielles signées par des ouvriers licenciés ainsi que des lettres d'avertissement envoyées aux ouvriers syndiqués de Birleşik Metal-İş̧ par leurs employeurs dans lesquelles ces derniers les menacent de les licencier s'ils refusent de changer de syndicat. Ils ont également mis en ligne sur des réseaux sociaux tels YouTube et Facebook des vidéos sous-titrées en anglais, enregistrées par le système de vidéosurveillance de l'entreprise, dans lesquelles sont donnés à voir à la fois des exemples d'insultes et d'humiliations que les employeurs font subir à leurs ouvriers, et la violence physique exercée contre certains ouvriers syndiqués du Birleşik Metal-İşs.

30. Emek Dünyasi (2012), « Bosch işçilerine saldırı : 7 iş̧̧i yaralı » [Attaque contre les ouvriers de Bosch : 7 ouvriers blessés], 16 avril ; disponible sur http://www.emekdunyasi.net/ed/isci-sendika/17106-bosch-iscilerine-demircubuklarla-saldirdilar, consulté le $1^{\text {er }}$ décembre 2016.

31. Le permanent syndical chargé des relations internationales du Birleşik Metal-İş est un jeune militant engagé également au sein d'un parti politique socialiste depuis le lycée. Né en 1982 à Istanbul, il est issu d'une famille de classe moyenne (mère femme au foyer, père commerçant). Il suit ses études dans un lycée anglophone ; il travaille au

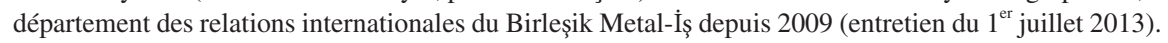

32. Le permanent syndical chargé des relations internationales de DİSK est un jeune militant engagé également au sein d'un parti politique socialiste depuis 1996. Il est né en 1981 à Edirne (à l'ouest de la Turquie) de parents fonctionnaires. Il est diplômé d'un lycée et d'une université anglophones d'Istanbul. Il travaille à DİSK comme permanent syndical chargé des relations internationales depuis 2007 (entretien du 15 février 2012). 
Le département des relations internationales du Birleşik Metal-Işs a ensuite envoyé de la part de son président une lettre à la Fédération française des travailleurs de la métallurgie de la Confédération générale du travail (CGT). Cette dernière a en retour publié une lettre de soutien au Birleşik Metal-I̦ş et à DİSK. Puis, le délégué syndical central CGT de Renault et le secrétaire général de la Fédération de la métallurgie ont adressé un courrier d' avertissement à l'attention du P.-D.G. de Renault et du directeur général du siège de Renault en France, dans lequel ils leur demandent « d'intervenir immédiatement pour réintégrer, sur le champ, les salariés licenciés et laisser les travailleurs de cette usine choisir librement leur syndicat ». Ils ajoutent qu' « au vu de la gravité de tels agissements, [ils se verraient] contraints d'alerter l'opinion publique à défaut d'une prise en compte immédiate de [leurs] demandes ».

Suite à l'internationalisation de ce cas de discrimination syndicale et de violence physique, les ouvriers de Renault à Bursa ont été réaffectés à leur poste dès la fin du procès ; celui des ouvriers de Bosch est toujours en cours au tribunal du travail.

\section{La mobilisation au niveau local : le cas de la zone portuaire à Tuzla à Istanbul}

La mobilisation au niveau local est également l'une des principales stratégies de résistance mise en œuvre par les syndicats, notamment lorsque leurs ressources sont trop faibles pour leur permettre de s'imposer au niveau national.

Situé au bord de la mer de Marmara, Tuzla est le quartier où se situent les plus grands docks d'Istanbul. Deux syndicats de dockers sont en présence : Dok-Gemi-İ̧, affilié à Türk-İs, et Limter-İs, affilié à DİSK. Sous le gouvernement AKP, Limterİşait l'objet d'actions de «marginalisation » et de « criminalisation ${ }^{33}$ » parce qu'il a organisé des manifestations pour protester contre les nombreux accidents du travail survenus dans les docks, dont certains ont eu pour conséquence la mort d'ouvriers. Zafer Çağlayan, ministre de l'Industrie au moment des faits en 2008, déclare que ces accidents de travail ont été provoqués par « des ennemis extérieurs de la Turquie, qui [veulent] empêcher le progrès de la Turquie dans le secteur de la construction navale et dans le commerce maritime ${ }^{34} »$.

Dok-Gemi-İşadopte le même type de discours (AKDEMir, OdMAN, 2008). C'est ainsi que son président, Necip Nalbantoğlu, explique au quotidien Vira Haber le 2 mars 2008 :

« Ils [les militants de Limter-İş] ont organisé une grande manifestation ici hier. Il y avait des représentants des partis politiques, des syndicats et d'autres groupes. Il y avait aussi des syndicalistes et des journalistes allemands. La Turquie est au cinquième rang mondial aujourd'hui dans le commerce maritime et l'Allemagne est au quatrième. Si nous parvenons à progresser autant en 2008 qu'en 2007, nous

33. Entretien avec le secrétaire général du Limter-İș Hakkı Demiral, 28 décembre 2012.

34. Ayaz E. (2008), « Çağlayan'dan Tuzla'da 'dış mihrak mı' sorusu », [Çağlayan se pose la question : « Est-ce qu'il y a des "ennemis extérieurs" à Tuzla ? »], Hürriyet, 24 mai ; http://hurarsiv.hurriyet.com.tr/goster/printnews. aspx?DocID=9012520, consulté le 22 décembre 2016. 
pouvons dépasser l'Allemagne. C'est pourquoi les syndicalistes et les journalistes allemands sont venus soutenir la manifestation hier. Ce sont les syndicalistes du Limter-İș qui les ont invités. »

De son côté, lors d'un entretien qu'il nous a accordé le 17 septembre 2012, le président du Limter-İş, Kamber Saygıll, indique : « Les ouvriers et nos opposants nous stigmatisent comme des terroristes » tandis que le secrétaire général du Limter-İş, Hakkı Demiral, décrit la situation suivante :

«Certaines entreprises ont invité le Dok-Gemi-Iș pour y faire adhérer leurs ouvriers. Leur seul but est d'éviter que leurs ouvriers ne se syndiquent au Limter-i̇ş. Elles les forcent donc à adhérer au Dok-Gemi-İş afin qu'elles puissent ensuite négocier des conventions collectives favorables à leurs propres intérêts. »

(Entretien avec le secrétaire général du Limter-İş, Hakkı Demiral, 28 décembre 2012)

Limter-İș, dont le bureau central est à Tuzla et n'a pas d'autre branche en Turquie, n'a eu d'autre choix pour défendre ses positions que celui de construire des alliances avec d'autres acteurs locaux, en particulier avec des partis politiques. Ainsi, durant les années 2000, il se rapproche du Parti socialiste des opprimés (Ezilenlerin Sosyalist Partisi, ESP) au point que les deux structures opèrent une quasi-fusion. Au niveau local à Tuzla, elles mutualisent leurs ressources, leurs militants et leurs activités. Les dirigeants du Limter-İs deviennent tous membres de l'ESP. Le parti fait embaucher ses militants aux docks pour qu'ils puissent adhérer ensuite au Limter-Iș. Veli, dirigeant du Limter-İs, décrit le processus :

« Nous avons noté, au sein du parti, que les docks étaient un espace de lutte important pour la lutte révolutionnaire, des dizaines de dockers étaient morts au travail. Donc nous avons décidé que je commencerais à travailler dans les docks. J'ai adhéré au Limter-İş et notre lutte révolutionnaire partisane s'est poursuivie au sein des docks et du syndicat. »

(Entretien avec Veli, dirigeant du Limter-İş, 17 mars 2014)

En les mutualisant, l'alliance a permis à ces deux acteurs d'augmenter les ressources dont ils disposent en vue de lutter contre la discrimination syndicale et la marginalisation du Limter-İ̧s à Tuzla. Elle sert également à trouver des solutions pratiques aux problèmes des ouvriers syndiqués discriminés :

«Limter-İs fait ce que l'État doit faire. Il assure la régularisation du paiement des salaires. Nous faisons pression sur les employeurs pour qu'ils paient aux ouvriers les indemnités de préavis, de licenciement et d'ancienneté. Aussi, quand tu n'es pas tout seul à lutter pour une cause, cela te rend plus puissant. Les alliances avec les partis politiques sont indispensables pour notre travail syndical. »

(Entretien avec Oktay, dirigeant syndical, docker à Tuzla, 23 juillet 2013) 
La mutualisation des ressources permise par l'alliance entre l'ESP et le Limter-İş est pleinement mobilisée. Le secrétaire général du Limter-Iș, Demiral, explique, dans un entretien du 28 décembre 2012 :

« Ils [les militants de l'ESP] diffusent des informations concernant nos manifestations ; ils les publient sur leurs journaux et leur site internet, etha.com.tr. Ils envoient leurs militants aux manifestations que nous organisons contre notre marginalisation et les menaces de licenciement de nos membres. Parfois, les ouvriers ne veulent pas que les employeurs les voient protester devant l'entreprise ; donc ils ne participent pas aux manifestations après le boulot. La présence des militants du parti est donc très importante. »

Lors de nos observations sur les docks, nous avons effectivement noté que les militants de l'ESP prennent part aux manifestations du Limter-İşà Tuzla et que le syndicat utilise aussi bien les ressources matérielles du parti (bus, pancartes, banderoles) que ses ressources humaines (les militants). En échange, les militants de l'ESP ont la possibilité de discuter avec les ouvriers lors de déjeuners ou de pauses-café ayant lieu à l'usine et lors des réunions syndicales.

Nous avons donc vu que des alliances entre différents acteurs au niveau local permettent de construire des stratégies de résistance contre la discrimination syndicale. Certains syndicats discriminés unissent leurs forces à des partis politiques également marginalisés afin de proposer une alternative aux ouvriers qui travaillent à la fois dans des conditions précaires, sous la menace de licenciement et de violence physique et morale de la part d'acteurs - employeurs ou syndicats concurrents - mettant en œuvre des pratiques discriminatoires.

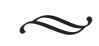

En Turquie, sous le gouvernement AKP, la discrimination syndicale s'articule autour de trois processus principaux : l'intervention(nisme) du gouvernement, qui a pour but de reconfigurer le champ syndical afin que celui-ci corresponde à ses orientations politiques ; la mutation de force ou le licenciement d'ouvriers syndiqués par leurs employeurs; le recours à la violence physique entre syndicats concurrents. Afin de résister à ces pratiques discriminatoires, certains syndicats ont mobilisé leurs réseaux à l'international et ont interpellé les syndicats des pays européens notamment, tandis que d'autres ont créé des alliances locales avec des partis politiques eux-mêmes en marge, dans un effort de mutualisation des ressources, des efforts et des luttes.

Les résultats de notre article contribuent aux études sur les différentes formes de discrimination et sur la transformation des répertoires d'action des syndicats. À partir du cas de la Turquie, ils permettent de dresser des premières pistes pour étudier les conséquences de l'intervention étatique, des politiques économiques néolibérales et de la précarisation du marché du travail non seulement sur les pratiques discriminatoires mais aussi sur la mobilisation, la syndicalisation et les conditions de travail des ouvriers. Ce faisant, ils alimentent la sociologie de l'action collective et des relations 
industrielles. Cette réflexion a permis de déceler les dynamiques intersectorielles et internationales du processus de précarisation du travail et de la consolidation démocratique en Turquie. Aussi, au terme de notre analyse, nous avons montré que les réformes juridiques entreprises pour respecter les conventions de l'OIT et les contraintes de la Commission européenne, dans le cadre du processus d'adhésion de la Turquie à l'Union européenne, n'empêchent pas, en pratique, la mise en œuvre de stratégies discriminatoires.

\section{BIBLIOGRAPHIE}

AKdemir N., Odman A. (2008), «Tuzla Tersaneler bölgesinde örülen ve üstü örtülen sınıfsallıklar » [Les relations de classe construites et cachées dans la zone des arsenaux à Tuzla], Toplum ve Bilim, $\mathrm{n}^{\circ}$ 113, pp. 49-89.

ATILGAN E., SANCAR Ü. (2009), Adalet biraz es geçiliyor. Demokratikleşme sürecinde hakimler ve savcllar [La justice est un peu négligée. Les juges et les procureurs dans le processus de démocratisation], Istanbul, Tesev (Türkiye Ekonomik ve Sosyal Etüdler Vakfi ; Turkish Economic and Social Studies Foundation).

BALKAN E., BALKAN N., ÖNCÜ A. (dir.) (2010), Neoliberalizm, islamcı sermayenin yükselişi ve AKP [Néolibéralisme, le renforcement du capital islamique et l'AKP], Istanbul, Yordam.

BECKER H. (1985), Outsiders : études de sociologie de la déviance, Paris, A.-M. Métailié.

Béroud S., Le CROM J.-P. Yon K. (coord.) (2012), « Représentativités syndicales, représentativités patronales. Règles juridiques et pratiques sociales. Introduction », Travail et Emploi, $\mathrm{n}^{\mathrm{o}} 131$, pp. $5-22$.

Bozarslan H. (2004), Histoire de la Turquie contemporaine, Paris, La Découverte.

BUĞRA A. (2010), Sinıftan sınıfa. Fabrika dışında çalışma manzaraları [De classe à classe. Le travail en dehors de l'usine], Istanbul, İletişim.

ÇALIŞMa ve Sosyal GüVENLIK BAKAnLIĞI [Ministère du Travail et de la Sécurité sociale] (2012), « 6536 sayılı Sendikalar ve toplu iş sözleşmesi kanunu [Loi des syndicats et des négociations collectives n ${ }^{\circ}$ 6536] », Resmi Gazete [Journal officiel] ; en ligne : http://www.resmigazete.gov. tr/eskiler/2012/11/20121107-1.htm, consulté le 23 décembre 2016.

Commission EuropéEnNe (2013), Turkey. 2013 Progress Report ; en ligne : http://ec.europa. eu/enlargement/pdf/key_documents/2013/package/brochures/turkey_2013.pdf, consulté le 5 décembre 2016.

DORRONSORO G. (dir.) (2005), La Turquie conteste. Mobilisations sociales et régime sécuritaire, Paris, Éditions du CNRS.

ERDINÇ I., VISIER C. (2017), « Droits sociaux et “dialogue social”, grandeur et décadence d'une notion », in Visier C. (dir.), La Turquie dans les années 2000, à parâtre. 
GouRISSE B. (2012), « Participation électorale, pénétration de l'État et violence armée dans la crise politique turque de la seconde moitié des années 1970. Contribution à l'analyse des crises politiques longues », Politix, nº 98, pp. 171-193.

GüLMEZ M. (2005), «Sendikal haklara ilişkin sözleşmelerin iç hukuka üstünlüğü ve yasalarımızdaki aykırılıklar » [La supériorité des conventions internationales sur le cadre juridique national et les incompatibilités dans notre législation], Çalışma ve Toplum, $\mathrm{n}^{\mathrm{o}}$ 1, pp. 11-56. INSEL A. (2008), « “Cet État n'est pas sans propriétaires !” Forces prétoriennes et autoritarisme en Turquie », in Dabène O., Geisser V., Massardier G. (dir.), Autoritarismes démocratiques et démocraties autoritaires au XXI ${ }^{e}$ siècle. Convergences Nord-Sud. Mélanges offerts à Michel Camau, Paris, La Découverte, pp. 133-153.

INSEL A. (2015), La Nouvelle Turquie d'Erdoğan. Du rêve démocratique à la dérive autoritaire, Paris, La Découverte.

KAYGisız İ. (2012), Kamuda Güvencesiz Çalışma [Travail précaire dans le secteur public], Ankara, Tüm Bel-Sen.

Koç Y. (2010), Türkiye İşçi Sınıfi Tarihi Osmanlı'dan 2010'a [L'histoire de la classe ouvrière Turque de l'État ottoman à 2010], Ankara, Epos.

LinZ J. J. (2006), Régimes totalitaires et autoritaires, Paris, Armand Colin.

ÖNGÜN E. (2009), « Temps et contretemps d'une européanisation. Le cas du mouvement contre l'exploitation aurifère en Égée du nord », European Journal of Turkish Studies, ${ }^{\circ} 9$; en ligne : http://ejts.revues.org/3733, consulté le 5 décembre 2016.

ÖZATalay K. C. (2010), Diversité des consciences ouvrières à l'ère des pragmatismes : l'ouvrier de l'État-nation versus l'ouvrier de la glocalisation : une étude sur le cas des ouvriers d'Isdemir, de Petkim et la TTK en Turquie, thèse de doctorat en sociologie, Paris, École des hautes études en sciences sociales.

Özveri M. (2012), Sendikal Haklar. 6356 Sayılı Sendikalar ve Toplu İş Sözleşmesi Yasası [Droits sociaux. Le code des syndicats et des conventions collectives numéro 6356], Istanbul, Birleşik Metal-İş.

PeCK J., Tickell A. (2007), “Conceptualizng Neoliberalism, Thinking Thatcherism”, in Leitner H., Peck J., Sheppard E. S. (eds.), Contesting Neoliberalism. Urban Frontiers, New York, London, Guilford Press, pp. 26-50.

Penissat É., Yon K. (2014), «Entraver l'action syndicale : des pratiques illégales aux techniques informelles de répression », in Observatoire de la discrimination et de la répression syndicales, Rapport 2014. De la discrimination individuelle à l'action collective. Propositions pour garantir le respect des droits syndicaux des salariés, chapitre III : «Un fait social difficile à mesurer », encadré 3, p. 57 ; en ligne : http://www.snetap-fsu.fr/IMG/pdf/rapport2014.pdf, consulté le 5 décembre 2016.

PORTA (della) D., TARrow S. (eds.) (2005), Transnational Protest and Global Activism, Lanham (Md.), Oxford, Rowman \& Littlefield.

Pulvar M. (2002), « La pratique du droit de la négociation en matière de conflits collectifs du travail en Martinique », in Virassamy G., Dauriac I., Edimo Nana F. et al. (dir.), Droit et 
pratiques syndicales en matière de conflits collectifs du travail. Actes du colloque des 18 et 19 décembre 2010 [organisé par le] Centre d'études et de recherches juridiques en droit des affaires, Faculté de droit et d'économie de la Martinique, Université des Antilles et de la Guyane, Paris, L'Harmattan, pp. 13-18.

SimÉANT J. (2009), « Transnationalisation/Internationalisation », in Fillieule O., Mathieu L., Péchu C. (dir.), Dictionnaire des mouvements sociaux, Paris, Presses de Sciences Po, pp. 554-564.

Sosyal HaKlar DerneĞi [Association des droits sociaux de la Turquie] (2012), Sosyal Hak İhlalleri Raporu [Rapport sur la violation des droits sociaux], Istanbul, SHD.

TANÖR B., YÜZBAşIOĞLU N. (2005), 1982 anayasasına göre Türk anayasa hukuku [Le droit constitutionnel turc selon la constitution de 1982], Istanbul, Beta.

WAGNER A.-C. (2004), « Syndicalistes européens. Les conditions sociales et institutionnelles de l'internationalisation des militants syndicaux », Actes de la recherche en sciences sociales, $\mathrm{n}^{\mathrm{o}} 155$, pp. 12-33. 